\title{
HAMILTON DECOMPOSITION OF COMPLETE BIPARTITE 3-UNIFORM HYPERGRAPHS
}

Jirimutu Jirimutu

College of Mathematics and Computer Science, Inner Mongolia for National University Tongliao 028043, P.R. China, jrmt@sina.com

Jul Wang

Department of Applied Mathematic, Dalian University of Technology, Dalian 116024, P.R. China.

Follow this and additional works at: https://jmstt.ntou.edu.tw/journal

Part of the Electrical and Computer Engineering Commons

\section{Recommended Citation}

Jirimutu, Jirimutu and Wang, Jul (2010) "HAMILTON DECOMPOSITION OF COMPLETE BIPARTITE 3-UNIFORM HYPERGRAPHS," Journal of Marine Science and Technology. Vol. 18: Iss. 5, Article 17.

DOI: $10.51400 / 2709-6998.1931$

Available at: https://jmstt.ntou.edu.tw/journal/vol18/iss5/17

This Research Article is brought to you for free and open access by Journal of Marine Science and Technology. It has been accepted for inclusion in Journal of Marine Science and Technology by an authorized editor of Journal of Marine Science and Technology. 
HAMILTON DECOMPOSITION OF COMPLETE BIPARTITE 3-UNIFORM HYPERGRAPHS

Acknowledgements

This work is supported by Education-funded of Inner Mongolia (Grant No.NJO4069).

This research article is available in Journal of Marine Science and Technology: https://jmstt.ntou.edu.tw/journal/ 


\title{
HAMILTON DECOMPOSITION OF COMPLETE BIPARTITE 3-UNIFORM HYPERGRAPHS
}

\author{
Jirimutu* and Jun Wang**
}

Key words: hypergraph, bipartite hypergraph, Hamilton cycle.

\begin{abstract}
The problem of finding a Hamilton decomposition of the complete 3-uniform hypergraph $K_{n}^{3}$ has been solved for $n$ 三 $2(\bmod 3), n \equiv 4(\bmod 6)[2], n \equiv 1(\bmod 6), n \equiv 0(\bmod 3)$ (for $K_{n}^{3}-I$, the complete 3-uniform hypergraph minus a 1 factor) [5]. In this paper, we give the concept of the bipartite hypergraphs and find a Hamilton decomposition of the complete bipartite hypergraph $K_{m, m}^{3}$ for $m$ be prime.
\end{abstract}

\section{INTRODUCTION}

A $k$-uniform hypergraph $H$ is a pair $(V, \varepsilon)$, where $V=\left\{v_{1}\right.$, $\left.v_{2}, \ldots, v_{n}\right\}$ is a set of $n$ vertices and $\varepsilon$ is a family of $k$-subset of $V$ called hyperedges. If $\varepsilon$ consists of all $k$-subset of $V$, then $H$ is a complete $k$-uniform hypergraph on $n$ vertices and is denoted by $K_{n}^{k}$. At the same time, we may refer to a vertex $v_{i} \in V$ as $v_{i+n}$. A cycle of length $l$ of $H$ is a sequence of the form $\left(v_{1}, e_{1}\right.$, $\left.v_{2}, e_{2}, \ldots, v_{l}, e_{l}, e_{1}\right)$, where $v_{1}, v_{2}, \ldots, v_{n}$ are distinct vertices, and $e_{1}, e_{2}, \ldots, e_{l}$ are $k$-edges of $H$, satisfying:

(i) $v_{i}, v_{i+1} \in e_{i} 1 \leq i \leq l$, where addition on the subscripts is modulo $n$, and

(ii) $e_{i} \neq e_{j}$ for $i \neq j$

This cycle is known as a Berge cycle, having been introduced by Berge in his book [1]. A Hamilton cycle of a hypergraph $H$ on $n$ vertices is a cycle of length $n$, and a Hamilton decomposition of $H$ is a partition of the hyperedges of $H$ into Hamilton cycles.

Definition 1. Let $H$ be a hypergraph on $V . H$ is called bipartite if $V$ can be partitioned into two subsets $V_{1}$ and $V_{2}$ such that $e \cap V_{1} \neq \phi$ and $e \bigcap V_{2} \neq \phi$ for any $e \in \varepsilon$. Furthermore, if $|e|=r$ for any $e \in \mathcal{E}$ then we call $H$ a bipartite $r$-uniform hypergraph, written $H^{r}\left(V_{1}, V_{2}\right)$. $H$ is called the complete bipartite

Paper submitted 09/03/09; revised 10/13/09; accepted 11/05/09. Author for correspondence: Jirimutu (e-mail: jrmt@sina.com).

*College of Mathematics and Computer Science, Inner Mongolia for National University Tongliao 028043, P.R. China.

**Department of Applied Mathematic, Dalian University of Technology, Dalian 116024, P.R. China. $r$-uniform hypergraph with vertex-set $V=V_{1} \cup V_{2}, V_{1} \cap V_{2}=$ $\phi$ if $\varepsilon=\left\{e: e \subseteq V,|e|=r\right.$ and $e \cap V_{i} \neq \phi$, for $\left.i=1,2\right\}$ and denoted it by $K^{r}\left(V_{1}, V_{2}\right)$ or $K_{n, m}^{r}$ when $\left|V_{1}\right|=n,\left|V_{2}\right|=m$.

A set of Hamilton cycles of $K^{r}{ }_{n, m}$, say $C_{1}, C_{2}, \ldots, C_{m}$ is called a Hamilton decomposition if $\bigcup_{i=1}^{m} \varepsilon\left(C_{i}\right)=\varepsilon\left(k_{n, m}^{r}\right)$ and $\varepsilon\left(C_{i}\right) \cap \varepsilon\left(C_{j}\right)=\phi$ for $i \neq j$.

In this paper, we give a Hamilton decomposition of complete bipartite hypergraph $K_{m, m}^{3}$ for $m$ being prime.

\section{RESULTS}

Let $m$ be a positive integer and let $D$ denote the set of all pairs $(k, r)$ of odd positive integers such that $k<r$.

Given a $(k, r) \in D$ and an integer $j$, define an edge $e_{j}(k, r)$ as follows:

(1) if $r \neq m$, $e_{j}(k, r)=\{j r, j r+k,(j+1) r\} \quad(\bmod 2 m) ;$

(2) if $r=m$ and $k$ is odd, $e_{j}(k, m)=\{j k, j k+m,(j+1) k+m\} \quad(\bmod 2 m) ;$ In both cases, define

$$
C(k, r)=\left\{e_{j}(k, r): j=0,1,2, \cdots, 2 m-1\right\}(\bmod 2 m)(1)
$$

Lemma 1 Let $m>3$ be a prime. Then, for any $(k, r) \in D$, $e_{j}(k, r)=e_{j^{\prime}}(k, r)$ if and only if $j \equiv j^{\prime}(\bmod 2 m)$.

Proof. By definition it is easily seen that $e_{j+2 m}(k, r)=e_{j}(k$, $r)$.

Suppose $e_{j}(k, r)=e_{j^{\prime}}(k, r)$ with $0 \leq j, j^{\prime} \leq 2 m-1$. Set $t=j^{\prime}-j$ and we consider two cases.

Case 1: $r \neq m$. We have that $\{j r, j r+k,(j+1) r\} \equiv\left\{j^{\prime} r, j^{\prime} r+\right.$ $\left.k,\left(j^{\prime}+1\right) r\right\}(\bmod 2 m)$, which implies that $\{0, k, r\} \equiv\left\{t r, t r+k_{1}\right.$, $(t+1) r\}(\bmod 2 m)$. If $t r \neq 0(\bmod 2 m)$ (equivalently, $t r+k \neq k$ $(\bmod 2 m)$ and $(t+1) r \neq r(\bmod 2 m))$, then,

(i) $t r \equiv k(\bmod 2 m), t r+k \equiv r(\bmod 2 m)$ and $(t+1) r \equiv 0(\bmod$ $2 m$ ); or

(ii) $t r \equiv r(\bmod 2 m), t r+k \equiv 0(\bmod 2 m)$ and $(t+1) r \equiv k$ $(\bmod 2 m)$. Both cases imply that $3 k \equiv 0(\bmod 2 m)$, a contradiction. It shows that $t r \equiv 0(\bmod 2 m)$. Recall that $r$ is odd and $r \neq m$, in other words, $r$ and $2 m$ are coprime, which implies that $j \equiv j^{\prime}(\bmod 2 m)$. 
Case 2: $r=m$. In this case we see that $m$ is a prime. We have that $\{j k, j k+m,(j+1) k+m\} \equiv\left\{j^{\prime} k, j^{\prime} k+m,\left(j^{\prime}+1\right) k+m\right\}$ $(\bmod 2 m)$, which implies that $\{0, m, k+m\} \equiv\{t k, t k+m,(t+$ $1) k+m\}(\bmod 2 m)$. If $t k \neq 0(\bmod 2 m)$ (equivalently, $t k+m \neq$ $m(\bmod 2 m)$ and $(t+1) k+m \neq k+m,(\bmod 2 m)$ then, (i) $t k \equiv m$ $(\bmod 2 m)$ and $(t+1) k+m \equiv 0(\bmod 2 m))$; or (ii) $t k \equiv k+m$ $(\bmod 2 m)$ and $t k+m \equiv 0(\bmod 2 m)$. Both cases imply that $k \equiv$ $0(\bmod 2 m)$, a contradiction. It shows that $t k \equiv 0(\bmod 2 m)$. Since $m$ is an odd prime and $k<m$ is an odd integer, we have that $k$ and $2 m$ are coprime, which implies that $j \equiv j^{\prime}(\bmod 2 m)$.

Lemma 2 Let $m>3$ be a prime and let $V_{1}=\{0,2, \ldots, 2 m-2\}$, $V_{2}=\{1,3, \ldots, 2 m-1\}$, and $K_{m, m}^{3}=K^{3}\left(V_{1}, V_{2}\right)$. Then the edge squence $C(k, r)$ defined in (1) and (2) is a Hamilton cycle of $K_{m, m}^{3}$.

Proof. By the definition of $e_{j}$, we see that, for every edge $e_{j}(k, r)$ of $C(k, r)$, exactly one of the following statements holds:

(1) $\left|e_{j} \cap V_{1}\right|=2$ and $\left|e_{j} \cap V_{2}\right|=1$, or

(2) $\left|e_{j} \cap V_{1}\right|=1$ and $\left|e_{j} \cap V_{2}\right|=2$.

From Lemma 1 it follows that $|C(k, r)|=2 m$, for each $(k, r) \in$ $D$. Note that if $r \neq m$, then $(j+2) r \neq j r(\bmod 2 m)$ for any integer $j$, and if $r=m$, then $m$ is prime and $(j+2) k+m \neq j k+m$ $(\bmod 2 m)$. From this it is easy to see that

$$
e_{j}(k, r) \bigcap e_{j+1}(k, r)= \begin{cases}(j+1) r & \text { if } r \neq m, \\ (j+1) k+m & \text { if } r=m .\end{cases}
$$

This proves that for each $(k, r) \in D, C(k, r)$ is a Hamilton cycle.

Lemma 3 Let $(k, r)$ and $\left(k^{\prime}, r^{\prime}\right)$ be two distinct elements of $D$. Then $C(k, r) \cap C\left(k^{\prime}, r^{\prime}\right)=\phi$.

Proof. Let us put the reduced residues modulo $2 m$ equidistantly and clockwise on a circle. Take three of them, say, $a$, $b$ and $c$. Then $\{a, b, c\} \in C(k, r)$ for some $(k, r) \in D$ if and only if the spaces among the three elements are in turn $k, r-k$ and $2 m-r$. Therefore, if $e_{j}(k, r)=e_{j^{\prime}}\left(k^{\prime}, r^{\prime}\right)$, then the cycle permutations $(k, r-k, 2 m-r)$ and $\left(k^{\prime}, r^{\prime}-k^{\prime}, 2 m-r^{\prime}\right)$ are identical. Note that there are only $r-k$ and $r^{\prime}-k^{\prime}$ are even. We therefore obtain that $k=k^{\prime}$ and $r-k=r^{\prime}-k^{\prime}$, which yields that $(k, r)=\left(k^{\prime}\right.$, $\left.r^{\prime}\right)$.

Theorem 4 Let $m>3, m$ be prime. Then $K_{m, m}^{3}=\bigcup_{(k, r) \in D} C(k, r)$ is a Hamilton decomposition.

Proof. Let $V_{1}=\{0,2, \ldots, 2 m-2\}, V_{2}=\{1,3, \ldots, 2 m-1\}$, and $K_{m, m}^{3}=K^{3}\left(V_{1}, V_{2}\right)$. By Lemma 2 , for any $(k, r) \in D, C(k, r)$ is a Hamilton cycle of $K^{3}\left(V_{1}, V_{2}\right)$. Therefore, in order to complete the proof it suffices to show that for each 3-element set $\{a, b, c\} \subseteq\{0,1, \ldots, 2 m-1\}$ with $\{a, b, c\} \cap V_{1} \neq \phi$ and $\{a, b, c\} \cap V_{2} \neq \phi$ there is a $(k, d) \in D$ and an integer $j$ such that $\{a, b, c\} \equiv e_{j}(k, r)(\bmod 2 m)$.

Without loss of generality we assume that $a<b<c$. Co- nsider $b-a, c-b$, and $2 m-c+a$. Since not all of them are even, while their sum is even, there are two among them are odd and one even. We label $b-a, c-b$, and $2 m-c+a$ as $k_{1}, k_{2}$ and $k_{3}$ such that $k_{1} \leq k_{3}$ are odd and $k_{2}$ is even. We now complete the proof by six cases.

Case 1: $\left(k_{1}, k_{2}, k_{3}\right)=(b-a, c-b, 2 m-c+a)$. In this case, put $k=k_{1}=b-a$ and $r=k_{1}+k_{2}=c+a$. If $r \neq m$, then $(r, 2 m)=$ 1 , there is a $j$ such that $a \equiv j r(\bmod 2 m)$, hence $b \equiv j r+k$ and $c$ $\equiv(j+1) r(\bmod 2 m)$, that is, $\{a, b, c\} \equiv e_{j}(k, r)(\bmod 2 m)$. If $r=$ $m$, then $(k, 2 m)=1$, there is a $j$ such that $a \equiv j k+m(\bmod 2 m)$, hence $b \equiv(j+1) k+m$ and $c \equiv j k(\bmod 2 m)$, that is, $\{a, b, c\} \equiv$ $e_{j}(k, m)(\bmod 2 m)$.

Case 2: $\left(k_{1}, k_{2}, k_{3}\right)=(b-a, 2 m-c+a, c-b)$. In this case, put $k=k_{3}=c-b$ and $r=k_{2}+k_{3}=2 m-c+a+c-b \equiv a-b$. If $r \neq m$, then $(r, 2 m)=1$, there is a $j$ such that $b \equiv j r(\bmod 2 m)$, hence $c \equiv j r+k$ and $a \equiv(j+1) r(\bmod 2 m)$, that is, $\{a, b, c\} \equiv$ $e_{j}(k, r)(\bmod 2 m)$. If $r=m$, then $(k, 2 m)=1$, there is a $j$ such that $b \equiv j k+m(\bmod 2 m)$, hence $c \equiv(j+1) k+m$ and $a \equiv j k$ $(\bmod 2 m)$, that is, $\{a, b, c\} \equiv e_{j}(k, m)(\bmod 2 m)$.

Case 3: $\left(k_{1}, k_{2}, k_{3}\right)=(c-b, b-a, 2 m-c+a)$. In this case, put $k=k_{3}=2 m-c+a$ and $r=k_{2}+k_{3}=2 m-c+a+b-a=b-$ $c$. The remainder is similar to Case 2 .

Case 4: $\left(k_{1}, k_{2}, k_{3}\right)=(c-b, 2 m-c+a, b-a)$. In this case, put $k=k_{1}=c-b$ and $r=k_{1}+k_{2}=c-b+2 m-c+a \equiv a-b$ $(\bmod 2 m)$. The remainder is similar to Case 1 .

Case 5: $\left(k_{1}, k_{2}, k_{3}\right)=(2 m-c+a, b-a, c-b)$. In this case, put $k=k_{1}=2 m-c+a$ and $r=k_{1}+k_{2}=2 m-c+a+b-a=a-$ $b(\bmod 2 m)$. The remainder is similar to Case 1 .

Case 6: $\left(k_{1}, k_{2}, k_{3}\right)=(2 m-c+a, c-b, b-a)$. In this case, put $k=k_{3}=b-a$ and $r=k_{2}+k_{3}=c-b+b-a=c-a$. The remainder is similar to Case 2 .

The proof is completed.

\section{ACKNOWLEDGMENTS}

This work is supported by Education-funded of Inner Mongolia (Grant No.NJO4069).

\section{REFERENCES}

1. Berge, C., Graphs and Hypergraphs, North-Holland, Amsterdam (1979).

2. Bermond, J. C., "Hamilton decompositions of graphs, directed graphs and hypergraphs," Annals of Discrete Mathematics, Vol. 3, pp. 21-28 (1978).

3. Jirimutu and Wang, J. F., "Hamiltonian decomposition of complete bipartite r-uniform hypergrahps," ACTA Mathematicae Applicatae Sinica, Vol. 17, pp. 563-566 (2001).

4. Katona, G. Y. and Kierstead, H. A., "Hamiltonian chains in hypergraphs," Journal of Graph Theory, Vol. 30, pp. 205-212 (1999).

5. Verrall, H., "Hamilton decompositions of complete 3-uniform hypergraphs," Discrete Mathematics, Vol. 132, pp. 333-348 (1994). 